\title{
Intramolecular hydrogen bond: Can it be part of the basis set of valence internal coordinates in normal mode analysis?
}

\author{
SARVESH KUMAR PANDEY ${ }^{\mathrm{a}}$, PRASANTA DAS ${ }^{\mathrm{b}}$, PUSPENDU K DAS ${ }^{\mathrm{b}}$, ELANGANNAN \\ ARUNAN $^{\mathrm{b}}$ and SADASIVAM MANOGARAN ${ }^{\mathrm{a}, *}$ \\ a Department of Chemistry, Indian Institute of Technology, Kanpur 208 016, India \\ ${ }^{b}$ Department of Inorganic and Physical Chemistry, Indian Institute of Science, Bangalore 560 012, India \\ e-mail: arunan@ipc.iisc.ernet.in; sm@iitk.ac.in
}

MS received 31 July 2014; revised 28 February 2015; accepted 28 February 2015

\begin{abstract}
It has been shown earlier ${ }^{1}$ that the relaxed force constants (RFCs) could be used as a measure of bond strength only when the bonds form a part of the complete valence internal coordinates (VIC) basis. However, if the bond is not a part of the complete VIC basis, its RFC is not necessarily a measure of bond strength. Sometimes, it is possible to have a complete VIC basis that does not contain the intramolecular hydrogen bond (IMHB) as part of the basis. This means the RFC of IMHB is not necessarily a measure of bond strength. However, we know that IMHB is a weak bond and hence its RFC has to be a measure of bond strength. We resolve this problem of IMHB not being part of the complete basis by postulating 'equivalent' basis sets where IMHB is part of the basis at least in one of the equivalent sets of VIC. As long as a given IMHB appears in one of the equivalent complete VIC basis sets, its RFC could be used as a measure of bond strength parameter.
\end{abstract}

Keywords. Hydrogen bond; intramolecular; relaxed force constant; normal mode analysis; bond strength parameter.

\section{Introduction}

The advantages of the compliance constants (the inverse of the force constant matrix elements) over the regular force constants were addressed by several authors. ${ }^{1-6}$ The relaxed force constant (RFC), reciprocal of the diagonal compliance matrix element, which was first introduced by Jones, ${ }^{7}$ was realized as a "more chemically meaningful bond strength parameter than the regular (force) constant." 8 Grunenberg explicitly showed this for covalent bonds in a series of papers and also extended the idea from covalent to noncovalent interactions like hydrogen bonding. ${ }^{5}$ However, it was pointed out that the RFCs of several nonbonded pairs have similar values as bonded pairs in several molecules. ${ }^{9}{ }^{10}$ Hence, to use the RFCs as a measure of bond strength parameter we have to separate the bonded atom pairs from the nonbonded ones. The RFCs are the measure of bond strength for the bonded atom pairs alone and not necessarily for nonbonded atom pairs. ${ }^{1}$

In a recent paper, ${ }^{1}$ we showed that if we use the valence internal coordinates (VIC) as the basis, the bonded pairs appear as stretching coordinates in VIC.

\footnotetext{
*For correspondence
}

In the case of dimers, there should be connecting bonds between monomers, which could be identified as bonded pairs in the dimer. The idea could be extended to larger clusters. Thus, the intermolecular hydrogen bonds will be part of the basis in VIC. ${ }^{1}$ The intramolecular hydrogen bonds need not be a member in the complete basis set of VIC defined in the standard normal mode analysis. Here we address this problem by defining 'equivalent' VIC basis sets by choosing intramolecular H-bonds (IMHBs) as part of atleast one basis. Since the complete basis sets are equivalent, the RFCs of bonds in all equivalent basis sets could be used as bond strength parameters.

\section{Methodology}

A polyatomic molecule having $N$ atoms has $3 N-6 / 3 N-$ 5 vibrational degrees of freedom, depending on whether it is nonlinear or linear. As a result, we have to choose $3 N-6 / 3 N-5$ suitable coordinates to address the vibrational problem. However, in most cases, the numbers of VIC are more than the number of vibrational degrees of freedom. As a result, we have to make the basis set linearly independent by making suitable linear combinations of VIC. We defined a complete basis set as 
the minimum number of required VIC to describe the normal modes as completely as possible. ${ }^{1}$ Since the normal modes depend on the symmetry of the molecule, this definition automatically includes all the VICs required to maintain the symmetry of a molecule. For example, in $\mathrm{BF}_{3}$, although three angles are linearly dependent, we need all of them to retain the $D_{3 h}$ symmetry in the complete basis set.

If we draw the normal mode pictures obtained from the mass-weighted cartesian force constants, the stretching frequency modes clearly indicate all the covalent bonds. ${ }^{1}$ The covalent bonds represent regions of electron density in between the bonded atoms, and the electron density is invariant. As a result, all the covalent bonds should be part of all the equivalent complete sets. Whenever redundancies are involved, usually in angles and torsions, there is no unique way to choose the angles and/or torsions as members of the

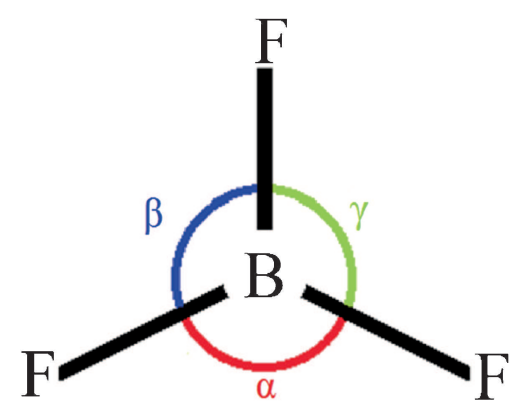

Set $1^{12}$

$S_{1}=\frac{1}{\sqrt{6}}(2 \alpha-\beta-\gamma)$

$S_{2}=\frac{1}{\sqrt{2}}(\beta-\gamma)$

Set $2^{13}$

$S_{2}=\frac{1}{\sqrt{2}}(\beta+\gamma)$;

$S_{2}=\frac{1}{\sqrt{2}}(\beta-\gamma)$

Set $3^{14}$

$\mathrm{S}_{1}=\alpha$;

$S_{2}=\frac{1}{\sqrt{2}}(\beta-\gamma)$

(normalized)

Set $4^{15}$

$\mathrm{S}_{1}=\alpha$

$S_{2}=\frac{1}{2}(\beta-\gamma)$

(unnormalized)

Figure 1. Equivalent sets representing the 3 angles in $\mathrm{BF}_{3}$.
VIC basis. For example, the three angles in $\mathrm{BF}_{3}$ are handled in different ways in the literature, as shown in figure $1 .^{11-15}$ Similarly, the 18 angles in benzene are handled in different ways in the literature, as shown in figure $2 .{ }^{16-18}$

In all the coordinate systems shown in figures 1 or 2 , the different sets produce the same set of normal modes of $\mathrm{BF}_{3}$ or benzene. Hence, they are equivalent sets. The equivalent basis sets are the sets of complete VICs giving the same set of normal modes for a given molecule.

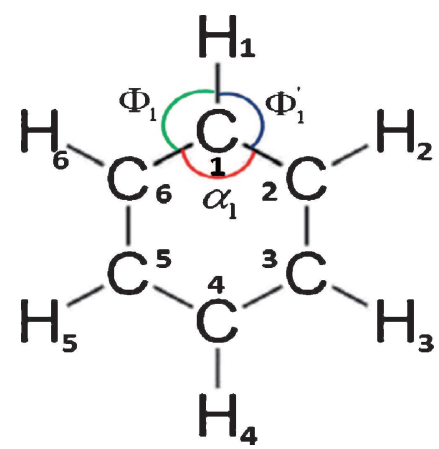

Set $1^{16}$

C-H rocking: $\beta_{1}, \beta_{2}, \beta_{3}, \beta_{4}, \beta_{5}, \beta_{6}$ where $\beta_{\mathrm{i}}=\frac{1}{2}\left(\Phi_{i}-\Phi_{i}^{\prime}\right)$ unnormalized ;

or,

$\beta_{\mathrm{i}}=\frac{1}{\sqrt{2}}\left(\Phi_{i}-\Phi_{i}^{\prime}\right)$ normalized $^{17}$.

In-plane deformation of ring, $B_{(1 u)}$ :

$\mathrm{q}_{19}=\frac{1}{\sqrt{6}}\left(\alpha_{1}-\alpha_{2}+\alpha_{3}-\alpha_{4}+\alpha_{5}-\alpha_{6}\right)$

In-plane deformation of ring, $E_{(2 g)}$ :

$\mathrm{q}_{20 \mathrm{a}}=\frac{1}{\sqrt{12}}\left(2 \alpha_{1}-\alpha_{2}-\alpha_{3}+2 \alpha_{4}-\alpha_{5}-\alpha_{6}\right)$

In-plane deformation of ring, $E_{(2 g)}$ :

$\mathrm{q}_{20 \mathrm{~b}}=\frac{1}{2}\left(\alpha_{2}-\alpha_{3}+\alpha_{5}-\alpha_{6}\right)$

Set $2^{18}$

CCC bend

$\mathrm{R}_{13}=\frac{1}{\sqrt{6}}\left(2 \alpha_{1}-\Phi_{1}-\Phi_{1}^{\prime}\right) ; \mathrm{R}_{19}=\frac{1}{\sqrt{2}}\left(\Phi_{1}-\Phi_{1}^{\prime}\right)$

$\mathrm{R}_{14}=\frac{1}{\sqrt{6}}\left(2 \alpha_{2}-\Phi_{2}-\Phi_{2}^{\prime}\right) ; \mathrm{R}_{20}=\frac{1}{\sqrt{2}}\left(\Phi_{2}-\Phi_{2}^{\prime}\right)$

$\mathrm{R}_{18}=\frac{1}{\sqrt{6}}\left(2 \alpha_{6}-\Phi_{6}-\Phi_{6}^{\prime}\right) ; \mathrm{R}_{24}=\frac{1}{\sqrt{2}}\left(\Phi_{6}-\Phi_{6}^{\prime}\right)$

Figure 2. Equivalent sets representing the 18 angles around the carbon atoms in $\mathrm{C}_{6} \mathrm{H}_{6}$. 
In the present work, equivalent VICs are defined for oxalic acid, 1,4-butanediol, and 1,2-ethanediol, where the IMHB is part of one of the equivalent basis sets. The RFCs and the normal modes are computed for each molecule using the equivalent VIC. The calculated normal modes show that the different VIC basis sets are indeed equivalent and the RFCs give the strength of IMHB in each system. For electronic structure calculations, Gaussian $09^{19}$ was used and the RFCs were calculated using a locally developed computer

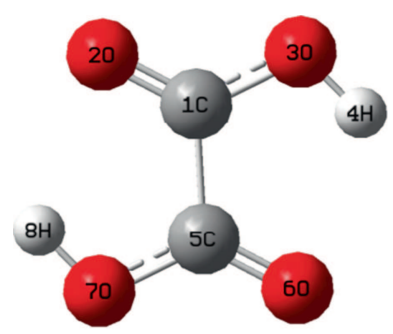

Bonds: 7 (covalent bonds indicates

Local* by the connecting lines)

Angles: $8(2 \angle \mathrm{COH}, 3$ angles around each $\mathrm{C} \times 2$ )

Out of plane: $2(2>\mathrm{C}=\mathrm{O})$

Torsions: 3 ( $\tau$ C-C, $2 \tau$ C-O)

7

6

2

3

Figure 3. VIC of Oxalic Acid without H-bonds. (*Local coordinates are $3 \mathrm{~N}-6$ linear combinations of VIC obtained as non-zero eigen vectors of suitable internal G-matrix ${ }^{6}$ ).

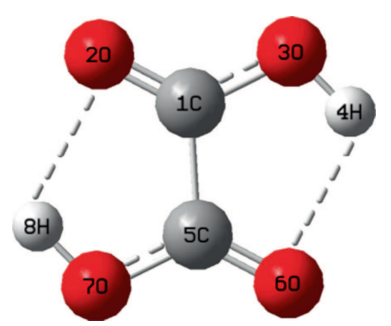

Bonds: 9 (covalent bond:7,

Local*

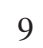

H bonds: 2)

Angles: 10 (each ring has 5 internal angles $\times 2$ )

Out of plane: 0

Torsions: 12 (each ring has 5

torsion $\times 2+2$ connecting the rings)

Figure 4. VIC Oxalic Acid with H-bonds. (*Local coordinates are 3N-6 linear combinations of VIC obtained as non-zero eigen vectors of suitable internal G-matrix ${ }^{6}$ ). program based on UMAT. ${ }^{20}$ All the optimized geometries of conformers are verified for the minima in potential energy surface by doing frequency calculations.

\section{Results and Discussion}

The main idea here is that the IMHB can be chosen as part of the VIC in at least one of the equivalent basis

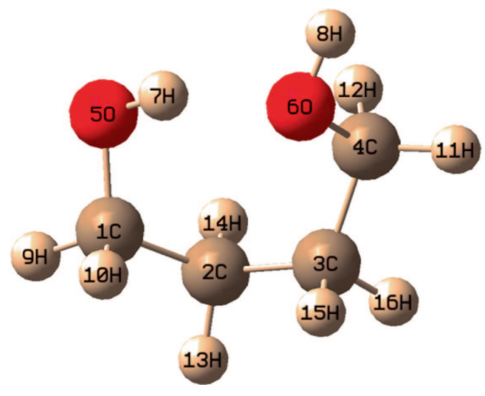

Bonds: 15 (all covalent bonds

Local* connected as lines)

Angles: $26(2 \angle \mathrm{COH}+6$ angles around each $\mathrm{C} \times 4)$

Torsions: $\mathbf{5}(3 \tau \mathrm{CC}+2 \tau \mathrm{CO})$

Figure 5. VIC of 1,4-Butanediol without H-bond. (*Local coordinates are $3 \mathrm{~N}-6$ linear combinations of VIC obtained as non-zero eigen vectors of suitable internal G-matrix ${ }^{6}$ ).

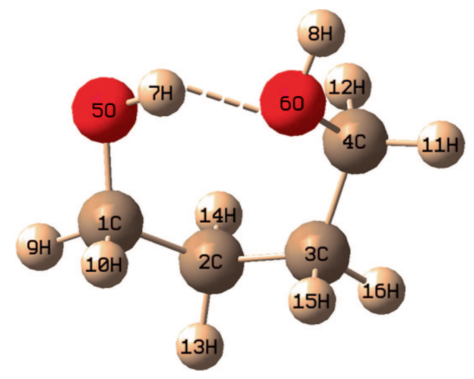

Bonds: 16 (covalent bonds: 15

Local* $+1 \mathrm{H}$ bond) Angles: 24 (6 angles

16 around each $\mathrm{C} \times 4$ ) Angles: 5+Torsions : 4 $[2 \angle \mathrm{COH}+\angle \mathrm{OHO}(576)+\angle \mathrm{HOC}(764)$ $+\angle \mathrm{HOH}(768)+\tau \mathrm{C}-\mathrm{O}(15)+\tau \mathrm{O}-\mathrm{H}(57)$ $+\tau \mathrm{O} \cdots \mathrm{H}(76)+\tau \mathrm{C}-\mathrm{O}(64)]$

6

Figure 6. VIC of 1,4-Butanediol with H-bond. (*Local coordinates are $3 \mathrm{~N}-6$ linear combinations of VIC obtained as non-zero eigen vectors of suitable internal G-matrix ${ }^{6}$ ). 
sets. As a result, the IMHB also can be included as a bonded pair in the VIC. The IMHB structure usually contains a ring as compared to the structure without the IMHB. Since the standard selection of VIC for a ring and an open chain compound is well documented in the literature, ${ }^{21}$ the complete VIC that describes the normal modes as completely as possible can easily be selected. In the present work, we have two equivalent basis sets for each molecule: one corresponding to the ring structure and the other corresponding to the open chain structure. We used two molecules, one planar (oxalic acid) and the other nonplanar (1,4-butanediol), as examples for this procedure. The two equivalent VIC are defined in figures 3 and 4 for oxalic acid and figures 5 and 6 for 1,4-butanediol, respectively. Using these VICs, we formed suitable 3N-6 linear combinations as local coordinates. These local coordinates are obtained as non-zero eigenvectors of a suitable internal G-matrix. ${ }^{6}$ The local coordinates thus formed are converted into symmetric coordinates and the normal mode analysis is done in symmetric coordinates to get the normal mode frequencies corresponding to different irreducible representations. All the normal mode frequencies and their corresponding eigenvectors matched for the equivalent basis sets. These results clearly show that the two basis sets for each of the molecule are really equivalent. The required results are given in the supplementary material (tables S1-S4). All the covalent bonds should be part of all of the equivalent basis sets for a given molecule.

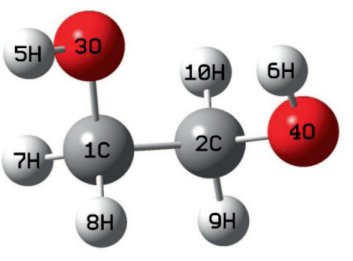

EG1

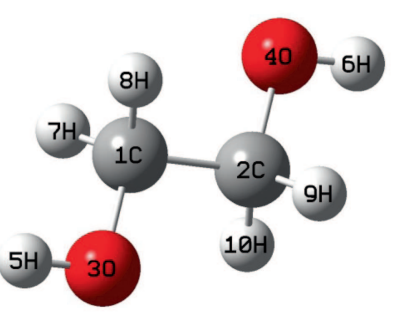

EG4

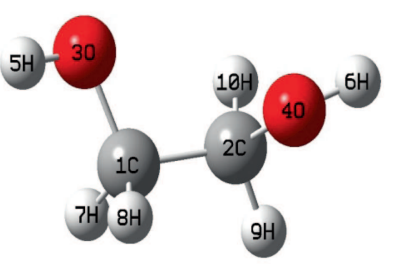

EG7

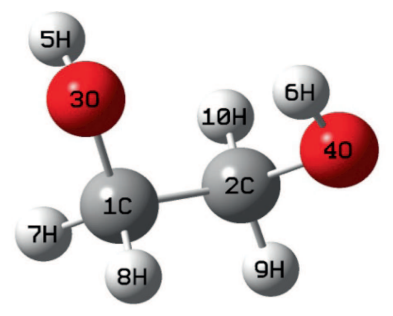

EG2

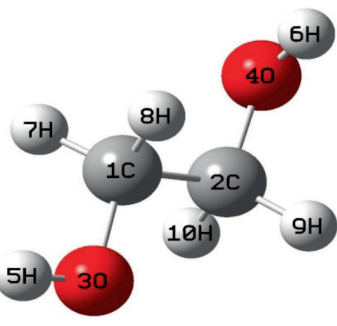

EG5

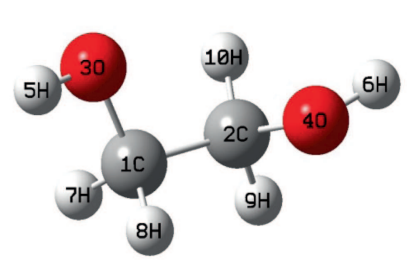

EG8

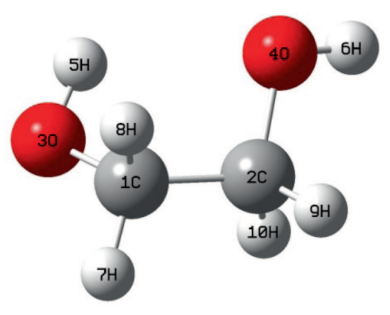

EG3 (EG1)
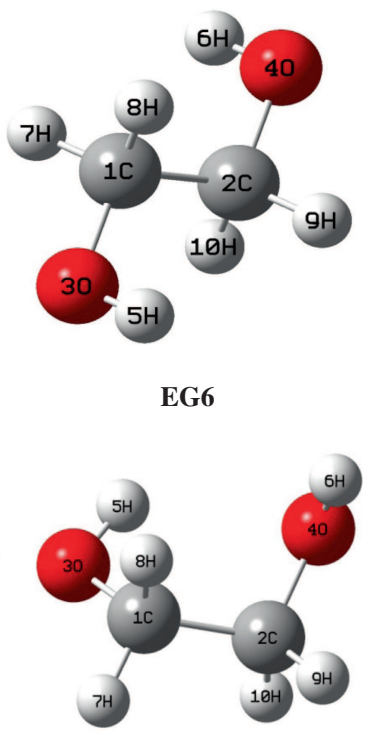

EG9 (EG2)

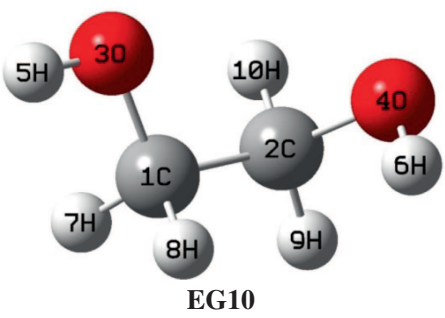

Figure 7. Optimized 1,2-ethanediol conformers at the MP2/cc-pvtz level. 
RFC values of IMHB were computed for oxalic acid and 1,4-butanediol using MP2/aug-cc-pvtz and the values are $0.21 \mathrm{mdyn} / \AA$ and $0.23 \mathrm{mdyn} / \AA \AA$, respectively. The IMHB bond distances are $2.096 \AA$ and $1.834 \AA$, respectively, at this level of theory. The $\angle \mathrm{CO} \ldots . \mathrm{H}$ and $\angle \mathrm{OH}$..... are $81^{\circ}, 118^{\circ}$ in oxalic acid and $103^{\circ}, 156^{\circ}$ in 1,4-butanediol, respectively. When the angles are close to $180^{\circ}$, usually the strongest bonds are formed. So the geometry explains that the IMHB of oxalic acid is weaker than that of 1,4-butanediol.

\subsection{Hydrogen bonding in 1,2-ethanediol}

Hydrogen bonding in 1,2-ethanediol received considerable attention in the literature. ${ }^{22-26}$ The full conformational space when scanned gave ten locally stable conformers: three hydrogen bonded and seven nonhydrogen bonded. ${ }^{22-24}$ The energy differences between these conformers are not very large. Overall, the results of different workers are consistent although the relative stability of different conformers is dependent on the method and basis sets used in the calculations as expected. We extended the calculations to higher basis sets MP2/cc-pvtz and MP2/aug-cc-pvtz. The results are shown in figures 7 and 8 and tables 1 and 2 . Our results are in agreement with the earlier results. The RFCs of the hydrogen bonded conformers are 0.09, 0.09, $0.09 \mathrm{mdyn} / \AA$ (MP2/cc-pvtz) and 0.08, 0.08, $0.01 \mathrm{mdyn} / \AA$ (MP2/aug-cc-pvtz), respectively. The low values of RFCs of the IMHB imply that the IMHB is extremely weak to be of any significance in 1,2ethanediol in agreement with the previous reports.

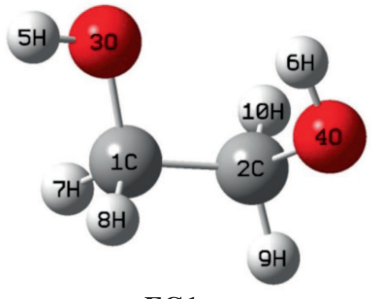

EG1

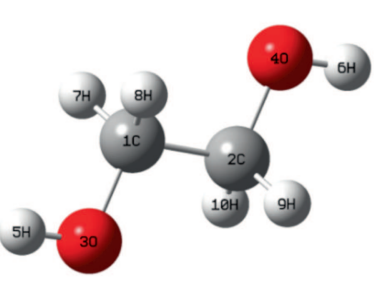

EG4

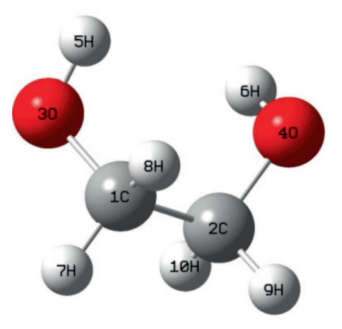

EG7 (EG3)

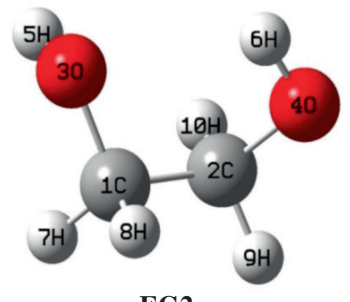

EG2

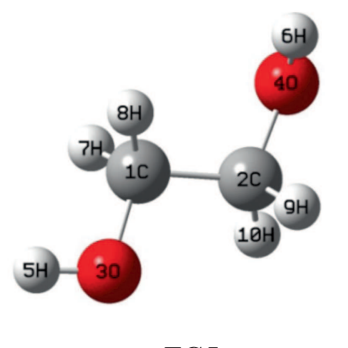

EG5

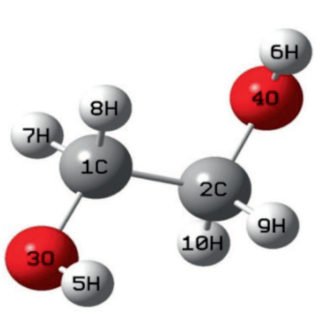

EG8
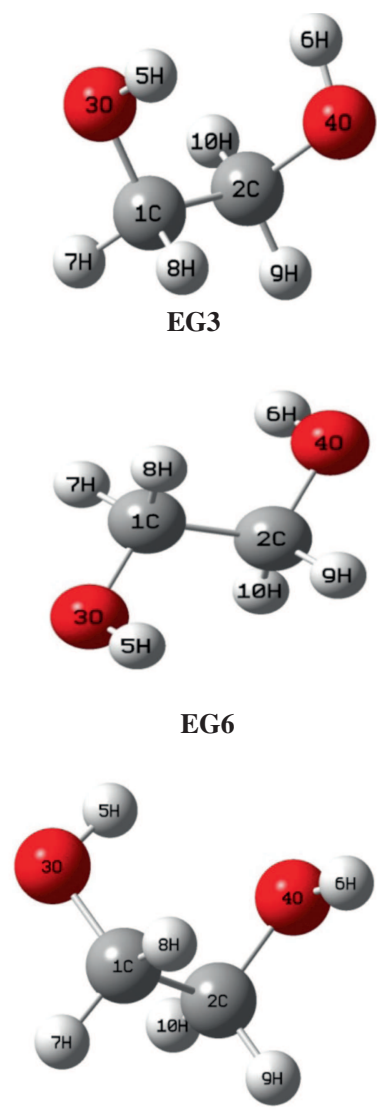

EG9 (EG2)

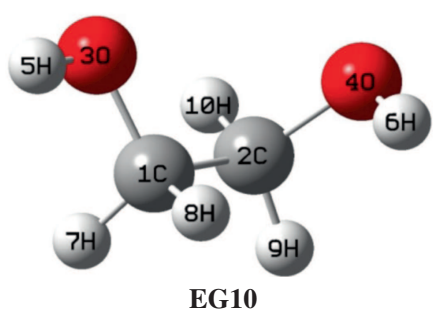

Figure 8. Optimized 1,2-ethanediol at the MP2/aug-cc-pvtz level. 
Table 1. Geometric parameters and relative energies of 1,2-ethanediol (MP2/cc-pvtz).

\begin{tabular}{|c|c|c|c|c|c|c|c|c|c|c|}
\hline Bonds** & EG1 & EG2 & EG3* & EG4 & EG5 & EG6 & EG7 & EG8 & EG9* & EG10 \\
\hline 112 & 1.502 & 1.506 & 1.502 & 1.503 & 1.508 & 1.512 & 1.498 & 1.512 & 1.506 & 1.503 \\
\hline 213 & 1.423 & 1.423 & 1.411 & 1.417 & 1.415 & 1.415 & 1.415 & 1.414 & 1.410 & 1.412 \\
\hline 324 & 1.411 & 1.410 & 1.422 & 1.417 & 1.415 & 1.415 & 1.415 & 1.414 & 1.423 & 1.412 \\
\hline 435 & 0.958 & 0.960 & 0.962 & 0.958 & 0.958 & 0.959 & 0.958 & 0.960 & 0.963 & 0.959 \\
\hline 546 & 0.962 & 0.963 & 0.958 & 0.958 & 0.959 & 0.959 & 0.958 & 0.960 & 0.960 & 0.960 \\
\hline 617 & 1.089 & 1.089 & 1.084 & 1.088 & 1.088 & 1.086 & 1.090 & 1.083 & 1.085 & 1.091 \\
\hline 718 & 1.088 & 1.084 & 1.090 & 1.088 & 1.091 & 1.088 & 1.088 & 1.092 & 1.093 & 1.093 \\
\hline 8210 & 1.090 & 1.093 & 1.088 & 1.088 & 1.083 & 1.088 & 1.088 & 1.083 & 1.084 & 1.084 \\
\hline 929 & 1.084 & 1.085 & 1.089 & 1.088 & 1.088 & 1.086 & 1.090 & 1.092 & 1.089 & 1.091 \\
\hline \multicolumn{11}{|l|}{ Angles** } \\
\hline 1312 & 105.8 & 110.3 & 111.0 & 106.8 & 106.9 & 111.9 & 108.3 & 111.9 & 110.8 & 108.6 \\
\hline 2421 & 111.0 & 110.8 & 105.8 & 106.8 & 111.6 & 111.9 & 108.3 & 111.9 & 110.3 & 113.7 \\
\hline 3531 & 108.5 & 107.6 & 105.1 & 107.9 & 108.2 & 107.6 & 108.1 & 107.8 & 105.2 & 108.0 \\
\hline 4642 & 105.1 & 105.2 & 108.5 & 107.9 & 107.7 & 107.6 & 108.1 & 107.8 & 107.6 & 107.6 \\
\hline 5712 & 110.1 & 110.9 & 109.9 & 108.9 & 108.8 & 109.5 & 108.7 & 109.1 & 110.2 & 108.2 \\
\hline 6812 & 109.2 & 109.5 & 108.9 & 108.9 & 109.2 & 109.3 & 108.3 & 109.7 & 109.3 & 109.1 \\
\hline 71021 & 108.9 & 109.3 & 109.2 & 108.9 & 109.2 & 109.3 & 108.3 & 109.1 & 109.5 & 108.8 \\
\hline 8921 & 109.9 & 110.2 & 110.1 & 108.9 & 109.3 & 109.5 & 108.7 & 109.7 & 110.9 & 108.4 \\
\hline \multicolumn{11}{|c|}{ Dihedral Angles** } \\
\hline 13124 & 60.7 & 56.3 & 60.5 & 180.0 & 179.1 & 180.0 & 73.3 & 176.2 & 56.3 & 64.3 \\
\hline 25312 & -166.7 & 72.9 & -49.5 & -180.0 & -175.8 & 69.2 & -166.6 & 64.7 & -42.4 & -179.1 \\
\hline 36421 & -49.7 & -42.4 & -167.3 & 180.0 & 70.4 & -69.2 & -166.6 & 64.7 & 72.7 & 56.1 \\
\hline 47124 & -178.9 & -179.4 & 179.0 & 59.0 & 57.8 & 62.8 & -165.8 & 58.4 & 175.2 & -174.3 \\
\hline 58124 & -58.8 & -59.4 & -62.3 & -59.0 & -60.1 & -55.0 & -47.9 & -59.4 & -66.4 & -57.3 \\
\hline 610213 & -62.2 & -66.5 & -59.0 & 59.0 & 61.6 & 55.0 & -47.9 & 58.4 & -59.4 & -53.5 \\
\hline 79213 & 179.2 & 175.1 & -179.0 & -59.0 & -56.2 & -62.8 & -165.8 & -59.4 & -179.4 & -170.9 \\
\hline \multicolumn{11}{|c|}{ Relative energies** } \\
\hline $\mathrm{HF}$ & 0.00 & 2.87 & 0.00 & 6.37 & 7.85 & 8.64 & 11.53 & 9.92 & 2.87 & 13.62 \\
\hline MP2 & 0.00 & 1.61 & 0.00 & 11.98 & 12.53 & 12.40 & 14.58 & 13.27 & 1.61 & 6.15 \\
\hline \multirow{2}{*}{\multicolumn{5}{|c|}{$\begin{array}{c}\text { Conformers } \\
\text { EG1 }\end{array}$}} & \multicolumn{3}{|c|}{ RFC Values for $(\mathrm{O}---\mathrm{H})$} & & & \\
\hline & & & & & \multicolumn{2}{|c|}{0.09} & & & & \\
\hline & \multirow{2}{*}{\multicolumn{2}{|c|}{$\begin{array}{l}\text { EG2 (EG9) } \\
\text { EG3 (EG1) }\end{array}$}} & & & \multirow{2}{*}{\multicolumn{2}{|c|}{$\begin{array}{l}0.09 \\
0.09\end{array}$}} & & & & \\
\hline & & & & & & & & & & \\
\hline
\end{tabular}

* After optimization EG3 goes to EG1 and EG9 goes to EG2 conformer.

** Bond lengths in Angstrom $(\AA)$, bond angles and dihedral angles in Degree $\left({ }^{\circ}\right)$, relative energies in $\mathrm{kJ} / \mathrm{mole}$ and $\mathrm{RFC}$ values in mdyn/ $\mathrm{A}$.

Table 2. Geometric parameters and relative energies of 1,2-ethanediol (MP2/aug-cc-pvtz).

\begin{tabular}{lcccccccccc}
\hline Bonds $^{* *}$ & EG1 & EG2 & EG3 & EG4 & EG5 & EG6 & EG7 ${ }^{*}$ & EG8 & EG9* & EG10 \\
\hline 112 & 1.502 & 1.505 & 1.506 & 1.503 & 1.508 & 1.512 & 1.506 & 1.512 & 1.505 & 1.503 \\
213 & 1.425 & 1.425 & 1.421 & 1.420 & 1.419 & 1.418 & 1.421 & 1.417 & 1.412 & 1.416 \\
324 & 1.414 & 1.412 & 1.421 & 1.420 & 1.418 & 1.418 & 1.421 & 1.417 & 1.425 & 1.415 \\
435 & 0.959 & 0.961 & 0.960 & 0.959 & 0.959 & 0.960 & 0.960 & 0.960 & 0.964 & 0.960 \\
546 & 0.963 & 0.964 & 0.960 & 0.959 & 0.960 & 0.960 & 0.960 & 0.960 & 0.961 & 0.960 \\
617 & 1.091 & 1.091 & 1.088 & 1.090 & 1.090 & 1.088 & 1.088 & 1.085 & 1.087 & 1.093 \\
718 & 1.090 & 1.086 & 1.090 & 1.090 & 1.093 & 1.090 & 1.090 & 1.093 & 1.095 & 1.094 \\
8210 & 1.092 & 1.095 & 1.090 & 1.090 & 1.085 & 1.090 & 1.090 & 1.085 & 1.086 & 1.086 \\
929 & 1.086 & 1.087 & 1.088 & 1.090 & 1.090 & 1.088 & 1.088 & 1.093 & 1.091 & 1.093
\end{tabular}


Table 2. (Continued)

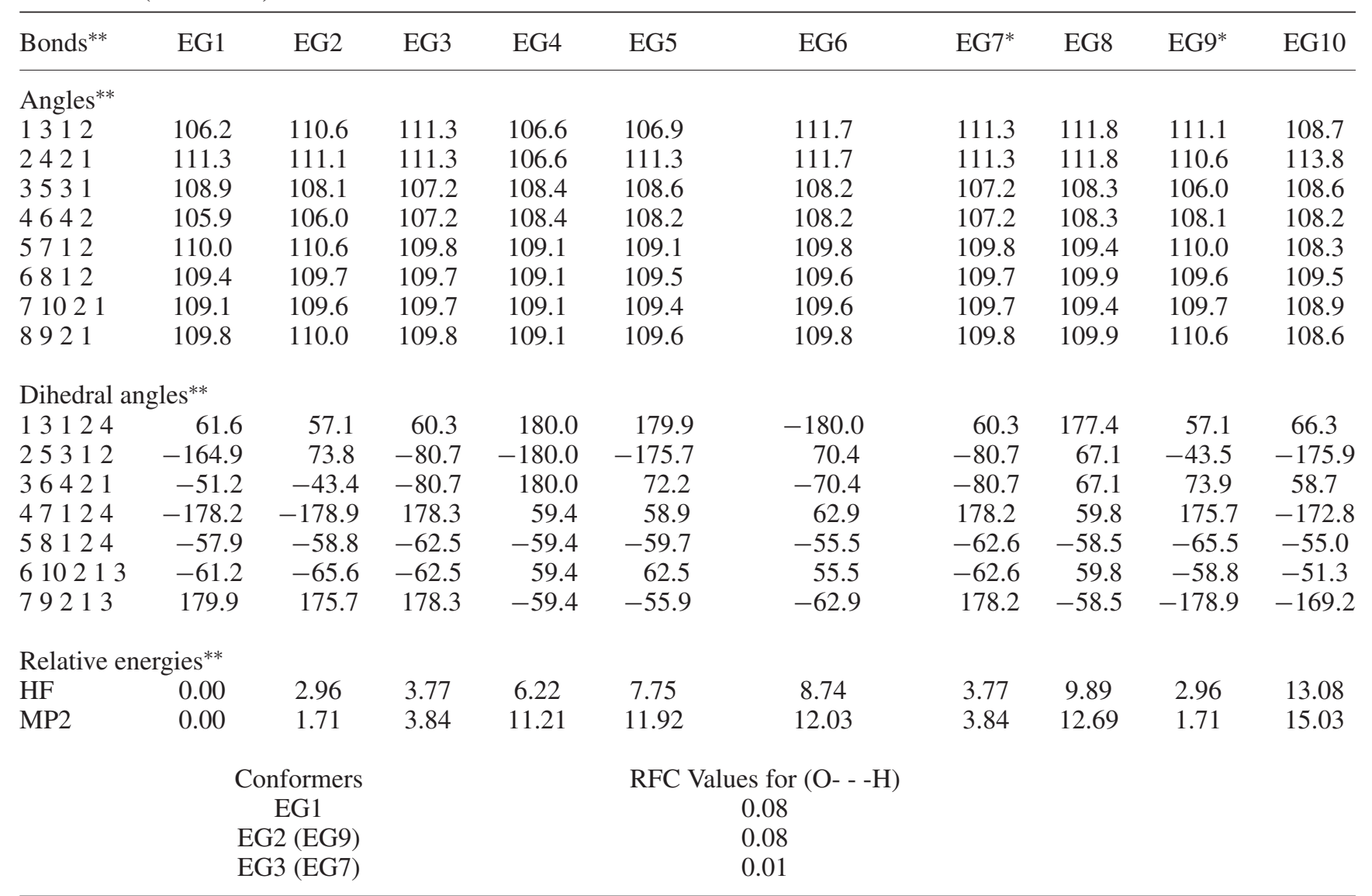

* After optimization EG7 goes to EG3 and EG9 goes to EG2 conformer.

** Bond lengths in Angstrom $(\AA)$, bond angles and dihedral angles in Degree $\left(^{\circ}\right)$, relative energies in $\mathrm{kJ} / \mathrm{mole}$ and $\mathrm{RFC}$ values in $\operatorname{mdyn} / \AA$.

\section{Conclusions}

The equivalent complete basis sets in VIC are defined in such a way that the intramolecular H-bond(s) is/are part of at least one set of VIC. As a result, their RFCs could be used as a measure of bond strength interaction. In 1,2-ethanediol, the RFC values indicate that the IMHB is extremely weak to be of any significance as predicted earlier.

\section{Supplementary Information}

Cartesian coordinates, internal coordinates, harmonic frequencies, local coordinates, and the normal modes in terms of cartesian displacement coordinates are given for oxalic acid and 1,4-butanediol with and without hydrogen bonding as supplementary material (tables S1-S4).

\section{Acknowledgements}

S.K.P. thanks the UGC, New Delhi, for a Research Fellowship and the authors thank Department of Science and Technology (DST), New Delhi, for supporting the Chemistry Department and IITK computational facilities.

\section{References}

1. Majumder M and Manogaran S 2013 J. Chem. Sci. 1259

2. Decius J C 1963 J. Chem. Phys. 38241

3. Cyvin S J 1968 In Molecular Vibrations and Mean Square Amplitudes (Amsterdam: Elsevier)

4. Jones L H and Swanson B I 1976 Acc. Chem. Res. 9128

5. Brandhorst K and Grunenberg J 2008 Chem. Soc. Rev. 371558 and references therein

6. Madhav M V and Manogaran S 2009 J. Chem. Phys. 131 174112

7. Jones L H 1970 Proc. Int. Conf. Coord. Chem. 13th [as given in Ref. 8]

8. Swanson B I 1976 J. Am. Chem. Soc. 983067

9. Baker J and Pulay P 2006 J. Am. Chem. Soc. 12811324

10. Baker J 2006 J. Chem. Phys. 125014103

11. Halgren T A 1990 J. Am. Chem. Soc. 1124714

12. Pulay P, Fogarasi G, Pang F and Boggs J E $1979 \mathrm{~J}$. Am. Chem. Soc. 1012550

13. Tan B T, Demaison J and Rudolph H D 1979 J. Mol. Spectrosc. 76104 
14. Mallinson P D and Nemes L 1976 J. Mol. Spectrosc. 59 470

15. Hollenstein H and Gunthard Hs H 1980 J. Mol. Spectrosc. 84457

16. Ozkabak A G and Goodman L 1987 J. Chem. Phys. 87 2564

17. Pulay P Fogarasi G and Boggs J E 1981 J. Chem. Phys. 743999

18. Torii H and Tasumi M 1995 J. Mol. Struct. 352/353 465

19. Gaussian 09, Revision D.01, Frisch M J, Trucks G W, Schlegel H B, Scuseria G E, Robb M A, Cheeseman J R, Scalmani G, Barone V, Mennucci B, Petersson G A, Nakatsuji H, Caricato M, X Li, Hratchian H P, Izmaylov A F, Bloino J, Zheng G, Sonnenberg J L, Hada M, Ehara M, Toyota K, Fukuda R, Hasegawa J, Ishida M, Nakajima T, Honda Y, Kitao O, Nakai H, Vreven T, Montgomery, Jr. J A, Peralta J E, Ogliaro F, Bearpark M, Heyd J J, Brothers E, Kudin K N, Staroverov V N, Kobayashi R, Normand J, Raghavachari K, Rendell A, Burant J C, Iyengar S S, Tomasi J, Cossi M, Rega N, Millam J M, Klene M, Knox J E, Cross J B, Bakken V,
Adamo C, Jaramillo J, Gomperts R, Stratmann R E, Yazyev O, Austin A J, Cammi R, Pomelli C, Ochterski J W, Martin R L, Morokuma K, Zakrzewski V G, Voth G A, Salvador P, Dannenberg J J, Dapprich S, Daniels A D, Farkas Ö, Foresman J B, Ortiz J V, Cioslowski J and Fox D J, Gaussian, Inc. Wallingford CT, 2009

20. McIntosh D F and Peterson M R, 1989 Program UMAT, QCPE576, University of Indiana, Bloomington, IN47405

21. Fogarasi G and Pulay P 1985 In Vibrational Spectra and Structure J R Durig (Ed.) Vol. 14 (Amsterdam: Elsevier) p. 193

22. Yeh T S, Chang Y P and Su T M 1994 J. Phys. Chem. 988921

23. Cramer C J and Truhlar D G 1994 J. Am. Chem. Soc. 1163892

24. Trindle C, Crum P and Douglass K 2003 J. Phys. Chem. 107A 6236

25. Chopra D, Guru Row T N, Arunan E and Klein R A 2010 J. Mol. Struct. 964126

26. Petterson K A, Stein R S, Drake M D and Roberts J D 2005 Magn. Reson. Chem. 43225 